\title{
Letter from the Board of Directors of Astronomy \& Astrophysics
}

\section{New A\&A memberships and scientific editorial structure for the Letters section}

At its meeting in Tartu, Estonia on 8 May 2004, the A\&A Board of Directors decided to grant observer status on the Board to Brazil, Chile, and Portugal (Sandqvist 2004, A\&A, 426, E15). Then on 6-7 May 2005, at its meeting in La Laguna, Spain, the Board of Directors admitted these three countries to full membership in A\&A, starting 1 January 2006.

The Letters Editor, Dr. P. Schneider, will complete his terms of service on 31 January 2006. A\&A is indebted to him for his thoughtful and competent editing over the past several years. As a consequence of his departure, the Board has decided to restructure the manner in which the Letters will be handled as of 1 January 2006. The Associate Editor-in-Chief, Dr. M. Walmsley, will also become Editor-in-Chief for the Letters, and he will forward the Letters to the appropriate topical Associate Editor to organize the reviewing process. Likewise, the Editor-in-Chief, Dr. C. Bertout, will become the Associate Letters-Editor-in-Chief. This change will permit a more specialized treatment of Letters in the future and also allow Letters to benefit from language editing. Hence, after 1 January 2006, manuscripts for Letters should be submitted via the A\&A Manuscript Management System (MMS) that is already in place for Main Journal submissions. Letters submitted before that will be handled by the current Letters Editor even after 1 January 2006.

\section{New Associate Editor positions}

Considering both the increased workload on the Associate Editors due to the above change and the continuing specialization of sub-fields in astronomy, the Board decided to open two new positions for Associate Editors, one specialized in Cosmology with a particular interest in theoretical aspects and the other in Observational Stellar Physics.

Applications are invited for these two new positions. The Associate Editors are expected to have a broad knowledge of astronomy and astrophysics and to have expertise in one of these two sub-fields. Candidates should have a strong record of published research in astronomy and astrophysics, should have experience as a referee and/or journal editor, and be prepared to commit the time needed to oversee the peer review of up to three hundred papers per year. Limited support for office equipment and secretarial help, as well as an annual indemnity, will be provided to the Associate Editors, and the initial term of appointment is three years.

Applicants should submit a curriculum vitae, a list of publications, and a concise covering letter that summarizes the candidate's qualifications and the reasons for seeking an Associate Editor position. The likelihood of support from the home institute for the task should also be discussed in the application. Applications should preferably be e-mailed or sent/faxed to the Chairman of the Board of Directors:

Dr. Georges Meynet

Geneva Observatory

1290 Sauverny

Switzerland

email: georges . meynet@obs .unige.ch

Fax:+41223792205

Applications received by 1 October 2005 will receive full consideration, while informal inquiries about the positions may be directed by e-mail to Georges Meynet.

On behalf of the Board of Directors Georges Meynet 